\title{
The statistics of atmospheric turbulence at Maunakea measured by RAVEN
}

\author{
Y. H. Ono ${ }^{a}$, Carlos M. Correia ${ }^{a}$, O. Lardière $^{b}$, D. R. Andersen ${ }^{b}$, S. Oya ${ }^{c}$, M. Akiyama ${ }^{d}$, D.

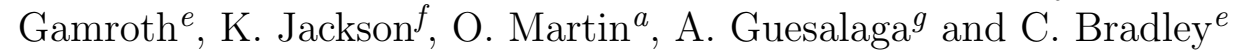 \\ ${ }^{a}$ Aix Marseille Université, CNRS, LAM (Laboratoire d'Astrophysique de Marseille) UMR \\ 7326, 13388, Marseille, France; \\ ${ }^{b}$ NRC-Herzberg, 5071 West Saanich Rd., Victoria, British Columbia, Canada; \\ ${ }^{c}$ TMT-J Project Office, NAOJ, 2-21-1 Osawa, Mitaka, Tokyo 181-8588, Japan; \\ ${ }^{d}$ Astronomical Institute, Tohoku University, 6-3 Aramaki, Aoba-ku, Sendai 980-8578, Japan; \\ ${ }^{e}$ Adaptive Optics Laboratory, University of Victoria, 3800 Finnerty Rd., Victoria V8P 5C2, \\ British Columbia, Canada; \\ ${ }^{f}$ Division of Engineering and Applied Science, California Institute of Technology, 1200 E. \\ California Boulevard MC 155-44, Pasadena, CA 91125, USA; \\ ${ }^{g}$ Pontificia Universidad Catlica de Chile, 4860 Vicuna Mackenna, Casilla 7820436, Santiago, \\ Chile;
}

\begin{abstract}
Prior statistical knowledge of the turbulence such as turbulence strength, layer altitudes and the outer scale is essential for atmospheric tomography in adaptive-optics (AO). These atmospheric parameters can be estimated from measurements of multiple Shack-Hartmann wave-front sensors (SH-WFSs) by the SLOpe Detection And Ranging (SLODAR). In this paper, we present the statistics of the vertical $C_{N}^{2}$ and the outer scale $\mathcal{L}_{0}$ at Maunakea in Hawaii estimated from 60 hours telemetry data in total from multiple SH-WFSs of RAVEN, which is an on-sky multi-object AO demonstrator tested on the Subaru telescope. The mean seeing during the RAVEN on-sky observations is $0.475 \mathrm{arcsec}$, and $55 \%$ turbulence is below $1.5 \mathrm{~km}$. The vertical profile of $C_{N}^{2}$ from the RAVEN SLODAR is consistent with the profiles from CFHT DIMM and MASS, and TMT site characterization.
\end{abstract}

Keywords: Atmospheric turbulence, RAVEN, SLODAR

\section{INTRODUCTION}

Prior statistical knowledge of the turbulence such as turbulence strength, layer altitudes, and wind speeds and directions is essential for atmospheric tomography in adaptive-optics $(\mathrm{AO}),{ }^{1}$ which reconstructs three-dimensional structure of the phase distortion caused by the atmospheric turbulence using multiple wave-front sensors (WFSs). These knowledge is also important for a post-processing such a performance evaluation of AO corrections ${ }^{2}$ and a Point Spread Function (PSF) reconstruction. ${ }^{3}$

Several techniques are proposed and tested on-sky at different sites to retrieve the statistical knowledge of the turbulence. ${ }^{4}$ The SLOpe Detection And Ranging (SLODAR) is a method commonly used to estimate the vertical profile of CN2 using multiple Shack-Hartmann WFSs (SH-WFS) by the triangulation between two stars. ${ }^{5-7}$ There are two kind of the SLODAR methods. The first approach uses the slope spatio cross-correlation method deconvolved by the slope auto-correlation and estimates the vertical $C_{N}^{2}$ from the intensities of peaks on the deconvolved spatio cross-correlation map, ${ }^{5}$ which is referred to as deconvolved-SLODAR in this paper. The advantage of the deconvolved-SLODAR is its model-independency. The second approach is recovering the vertical turbulence profile by fitting the theoretical spatio cross-correlation to the observed spatio cross-correlation, ${ }^{6,7}$ which is referred to as fitted-SLODAR. The fitted-SLODAR can also measure the vertical profile of the outer scale L0 by using the von Karman power spectrum model for the turbulence. ${ }^{8}$

Further author information: yoshito.ono@lam.fr 
In this paper, we show the turbulence statistics at Maunakea in Hawaii measured by RAVEN, ${ }^{9}$ which is a Multi-Object AO (MOAO) demonstrator installed on the Subaru telescope. RAVEN have successfully completed their on-sky engineering observations on the Subaru telescope. During the on-sky runs, we estimate the turbulence profile using the fitted-SLODAR assuming the same outer scale for all altitudes. In this paper, we reprocess the turbulence profiling offline for all telemetries from the on-sky runs using the fitted-SLODAR improved to estimate the outer scale at each altitude as well and to estimate the turbulence unsensed only by the spatio cross-correlation. Then, we provide statistics of the vertical profiles of $C_{N}^{2}$ and $\mathcal{L}_{0}$ at Maunakea. In Section 2, we explain our fitted-SLODAR method. Then, we present the vertical profiles of $C_{N}^{2}$ and the outer scale $\mathcal{L}_{0}$ in Section 3 and finally we conclude our results in Section 4.

\section{SLODAR METHOD}

Our fitted-SLODAR method is based on Butterley et al. $(2006)^{6}$ and Cortés et al. (2012), ${ }^{7}$ which estimates the vertical turbulence profile by fitting the theoretical correlations to the observed one. Here, we consider the case with two natural guide stars (NGSs) with the angular separation $\theta$, two corresponding SH-WFSs and $n_{\text {layer }}$ turbulence layers at different altitudes, as shown as Fig.1. The $x$-slope of the subaperture indexed as $(i, j)$ of WFS 1 is noted as $s_{i, j}^{x[1]}$. First, we subtract the temporal average and tip/tilt from a time series of measured slopes. Then, the spatio cross-correlation of $x$ - and $x$-slopes with the subaperture offset of $(\delta i, \delta j)$ between WFS 1 and WFS 2 is computed as ${ }^{6}$

$$
C^{x[1] x[2]}(\delta i, \delta j)=\frac{\left\langle\sum_{i, j} s_{i, j}^{x[1]} s_{i+\delta i, j+\delta j}^{x[2]}\right\rangle}{O(\delta i, \delta j)},
$$

where $O(\delta i, \delta j)$ is the number of the valid subaperture pairs with the offset of $(\delta i, \delta j), \sum_{i, j}$ denotes a summation for all valid subapertures and \langle\rangle denotes the average over the time series. The spatio cross-correlation map, noted as $\boldsymbol{C}^{\boldsymbol{x}[1] \boldsymbol{x}[2]}$, is given by concatenating the $C^{x[1] x[2]}(\delta i, \delta j)$ for all $(\delta i, \delta j)$. The $y-y, x-y$ and $y-x$ spatio cross-correlation between two WFSs can be given as well. The $x-x$ spatio auto-correlation for WFS 1 is given as

$$
A^{x[1]}(\delta i, \delta j)=\frac{\left\langle\sum_{i, j} s_{i, j}^{x[1]} s_{i+\delta i, j+\delta j}^{x[1]}\right\rangle}{O(\delta i, \delta j)} .
$$

The observed correlations are noted as $\boldsymbol{C}_{\mathrm{obs}}^{x[1] x[2]}$ and $\boldsymbol{A}_{\mathrm{obs}}^{x[1]}$, and the theoretical correlations are noted as $\boldsymbol{C}_{\mathrm{theo}}^{x[1] x[2]}$ and $\boldsymbol{A}_{\mathrm{theo}}^{x[1]}$. The theoretical spatio correlations are computed based on Butterley et al. $(2006)^{6}$ using the von Karman power spectra model, and these correlations are a function of the turbulence altitude $h_{k}$, Fried parameter $r_{0, k}$ and the outer scale $\mathcal{L}_{0, k}$. The vertical profiles of $r_{0}\left(\right.$ or $\left.C_{N}^{2}\right)$ and $L_{0}$ can be estimated by fitting the theoretical correlation maps to the observed correlation maps. The altitude resolution is fixed, and, hence, the free parameters for the fitting are $r_{0, k}$ and $\mathcal{L}_{0, k}$ at each altitude. We do not use the $x-y$ and $y-x$ correlations for the fitting because there is no strong feature on these correlation maps.

RAVEN uses three NGSs and, hence, there are three WFS pairs. Since the optimal altitude resolution for the SLODAR is determined by the angular separation of two NGSs, each NGS pair has a different altitude resolution. However, in order to perform the fitting simultaneously with all three pairs, we have to use the same altitude resolution for all pairs. In this study, we use the minimum altitude resolution in the three pairs. It means the oversampling for the other NGS pairs, but we can increase a signal-to-noise ratio simultaneously using three pairs.

We use both the spatio cross- and auto-correlation for the fitting. Although the vertical turbulence profile can be estimated from the spatio cross-correlation fitting, the maximum altitude measured by the spatio crosscorrelation is limited as $h_{\max }=\theta / d_{\max }$ as shown in Fig.1, where $d_{\max }$ is the maximum spatial separation in all valid subaperture pairs, and, hence, the turbulence above $h_{\max }$ can not be seen by the spatio cross-correlation, where we referred to this turbulence as unsensed turbulence. On the other hand, the spatio auto-correlation can measure the integrated $r_{0}$ and $\mathcal{L}_{0}$, including the unsensed turbulence. Using both the spatio cross- and autocorrelation, we can measure the total $r_{0}$ and $\mathcal{L}_{0}$ of the unsensed turbulence higher than $h_{\max }$, but the altitude of the unsensed turbulence can no be estimated. 


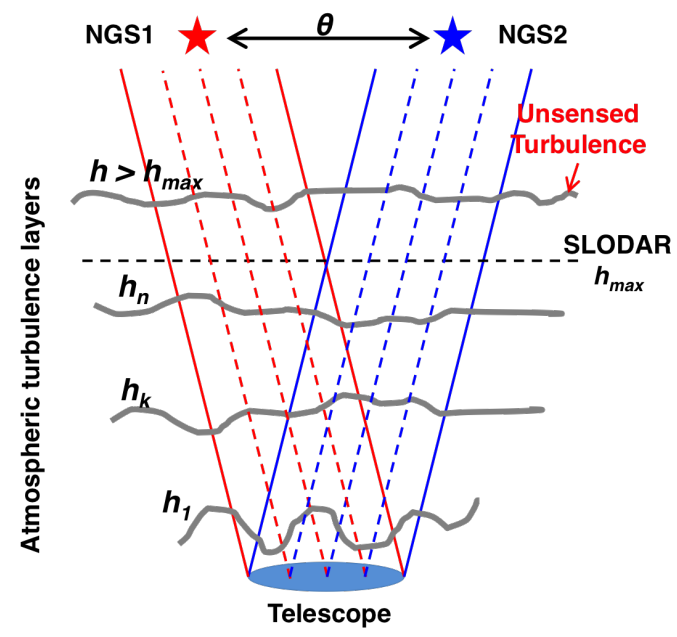

Figure 1. Schematic illustration of the SLODAR method. The SLODAR method estimates the vertical turbulence profile by a triangulation by 2 stars with the angular separation of $\theta$. The maximum altitude $h_{\text {mas }}$, which can be measured by the SLODAR, is limited depending on $\theta$.

The cost function for the spatio cross-correlation is given as

$$
\chi_{\text {cross }}^{2}=\left\|\sum_{p, q} \boldsymbol{M}_{\text {cross }}^{[p q]}\left[\begin{array}{cc}
\boldsymbol{\Sigma}_{\text {cross }}^{x[p] x[q]} & \mathbf{0} \\
\mathbf{0} & \boldsymbol{\Sigma}_{\text {cross }}^{y[p] y[q]}
\end{array}\right]^{-1}\left(\left[\begin{array}{l}
\boldsymbol{C}_{\text {obs }}^{x[p] x[q]} \\
\boldsymbol{C}_{\text {obs }}^{y[p] y[q]}
\end{array}\right]-\sum_{k}\left[\begin{array}{l}
\boldsymbol{C}_{\text {theo }}^{x[p] x[q]}\left(h_{k}, r_{0, k}, \mathcal{L}_{0, k}\right) \\
\boldsymbol{C}_{\text {theo }}^{y[p] y[q]}\left(h_{k}, r_{0, k}, \mathcal{L}_{0, k}\right)
\end{array}\right]\right)\right\|^{2},
$$

where $[p, q]=[1,2],[1,3]$ and $[2,3]$. The second matrix in Eq.(3) is a weighting matrix, and $\boldsymbol{\Sigma}_{\text {cross }}^{x[p] x[q]}$ is the inverse matrix of the temporal variance of the observed spatio cross-correlation over a time series. The temporal variance of the observed cross-correlation increases with the spatial offset between two subapertures due to the small number of the valid subaperture pairs, this is, the estimates of high altitude turbulences are affected more by the WFS measurement noise than those of the low altitude turbulence. Therefore, we reduce the weight of the correlation with large subapertures offset by the inverse temporal variance of the cross-correlation during the fitting process. Since the spatio cross-correlation peaks caused by the turbulence layers appear along the baseline of the 2 stars, we extract the spatial cross-correlations only around the baseline of NGSs by $\boldsymbol{M}_{\text {cross }}^{[p q]}$.

The cost function for the spatio auto-correlation is given as

$$
\begin{aligned}
\chi_{\text {auto }}^{2}= & \| \sum_{p} \boldsymbol{M}_{\text {auto }}^{[p]}\left[\begin{array}{cc}
\boldsymbol{\Sigma}_{\text {auto }}^{x[p]} & \mathbf{0} \\
\mathbf{0} & \boldsymbol{\Sigma}_{\text {auto }}^{y[p]}
\end{array}\right]^{-1} \\
& \left(\left[\begin{array}{l}
\boldsymbol{A}_{\text {obs }}^{x[p]} \\
\boldsymbol{A}_{\text {obs }}^{y[p]}
\end{array}\right]-\sum_{k}\left[\begin{array}{l}
\boldsymbol{A}_{\text {theo }}^{x[p]}\left(r_{0, k}, \mathcal{L}_{0, k}\right) \\
\boldsymbol{A}_{\text {theo }}^{y[p]}\left(r_{0, k}, \mathcal{L}_{0, k}\right)
\end{array}\right]-\left[\begin{array}{l}
\left.\boldsymbol{A}_{\text {theo }}^{x[p], \text { unsensed }}\left(r_{0}^{\text {unsensed }}, \mathcal{L}_{0}^{\text {unsensed }}\right)\right] \\
\boldsymbol{A}_{\text {theo }}^{y[p], \text { unsensed }}\left(r_{0}^{\text {unsensed }}, \mathcal{L}_{0}^{\text {unsensed }}\right)
\end{array}\right]\right) \|^{2},
\end{aligned}
$$

where $\boldsymbol{\Sigma}_{\text {auto }}^{x[p]}$ is the temporal variance of the observed auto-correlation over a time series and $\boldsymbol{M}_{\text {auto }}^{[p]}$ is a masking

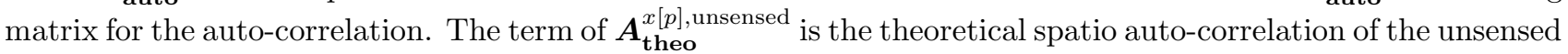
turbulences, which affects only the spatio auto-correlation.

The vertical profile of $r_{0}$ and $\mathcal{L}_{0}$ can be estimated by minimizing $\chi_{\text {cross }}^{2}$ and $\chi_{\text {auto }}^{2}$ with the LevenbergMarquardt algorithm.

\section{RESULTS}

RAVEN had three on-sky engineering runs on the $8 \mathrm{~m}$ Subaru telescope at the top of Maunakea, and we have in total 60 hours on-sky telemetry data from 12 nights. We reprocessed the telemetry with the SLODAR method 

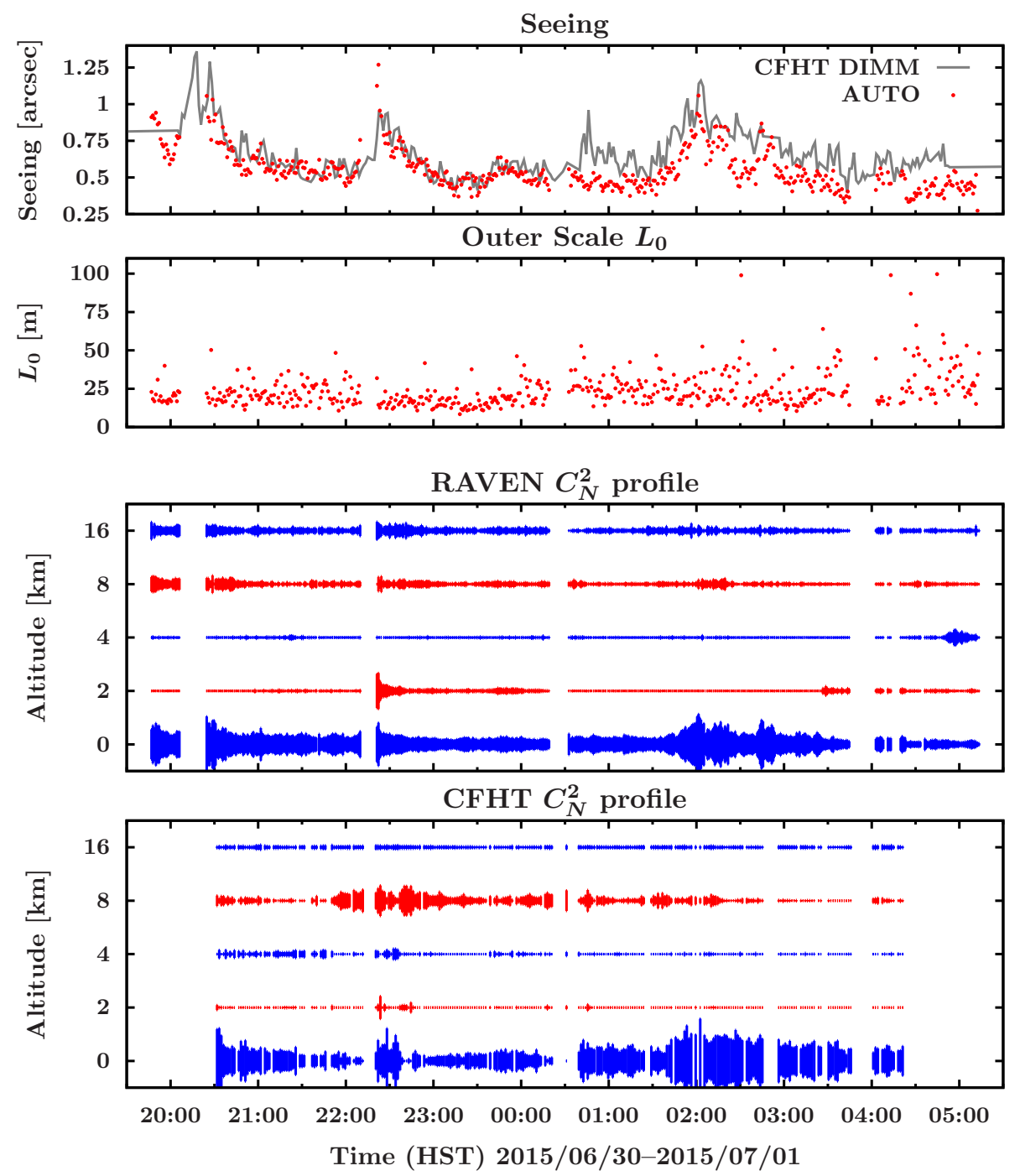

Figure 2. Top: Seeing profile on the night of June 30, 2015. The red plots represent the seeing estimated by the RAVEN SLODAR. The gray line shows the seeing profile from the CFHT DIMM. Second panel from the top: Outer scale profile on the same night, estimated by the RAVEN SLODAR. Third panel from the top: $C_{N}^{2}$ profile estimated by the RAVEN SLODAR. The profile is resampled to compare with the result of CFHT DIMM and MASS. The errorbar shows $C_{N}^{2}$ value at each altitude at each time. Bottom: Same as the third figure for $C_{N}^{2}$ profile estimated by the CFHT DIMM and MASS.

for all measurements and make the statistics of the vertical profiles of $C_{N}^{2}$ and $\mathcal{L}_{0}$. In the remainder of this paper, all estimates are scaled to values in the zenith direction. Each estimate is retrieved from 1 minute time series of the slope.

First, Fig.2 shows the estimated profiles of the integrated seeing, the integrated outer scale and $C_{N}^{2}$ at each altitude over one night of June 30, 2015. The total seeing and vertical $C_{N}^{2}$ profile, estimated by a Differential Image Motion Monitor (DIMM) and a Multi-Aperture Scintillation Sensor (MASS) located at the CanadaFrance-Hawaii Telescope $(\mathrm{CFHT})^{*}$, are also plotted on Fig. 2 as comparison. The seeing changes from 0.25 arcsec to 1.25 arcsec over the night, and the result from the RAVEN SLODAR is consistent with the seeing from the CFHT DIMM.

The outer scale is roughly $20 \mathrm{~m}$ with large variations. The similar integrated $\mathcal{L}_{0}$ are observed at different sites using different methods with large spatial scales of a few $100 \mathrm{~m}^{10,11}$ However, it is noted that the SLODAR

\footnotetext{
${ }^{*}$ http://mkwc.ifa.hawaii.edu/seeing
} 


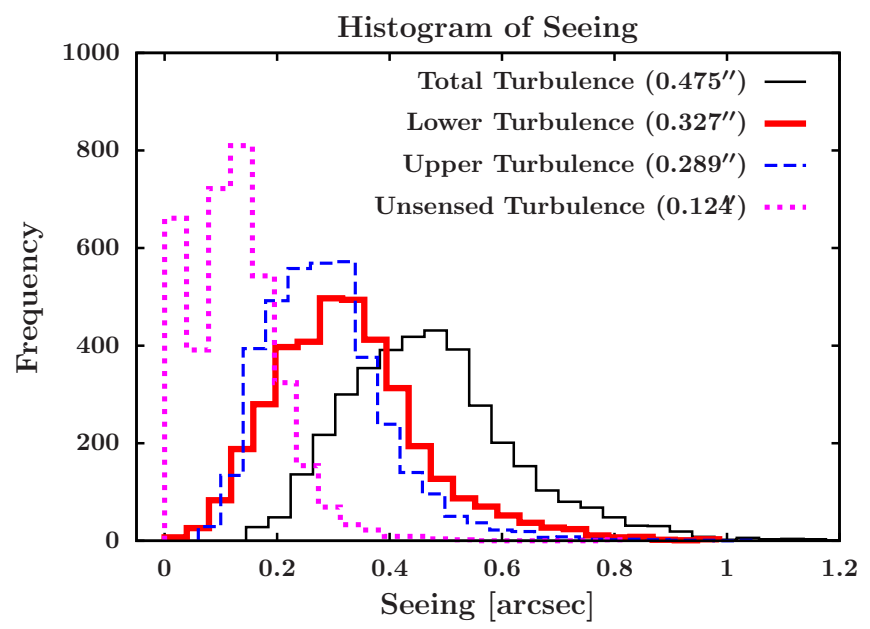

Figure 3. Histogram of seeing. The black line shows the total seeing. The red and blue lines show the seeing at the lower altitude $(h<1.5 \mathrm{~km})$ and the upper altitude $(h \geq 1.5 \mathrm{~km})$, respectively. The magenta line shows the histogram of the unsensed seeing. The mean values are 0.475 arcsec for the total, 0.327 arcsec for the lower altitude, 0.289 arcsec for the upper altitude and 0.124 arcsec for the unsensed turbulence.

method is not sensitive to the outer scale larger than the size of a telescope aperture diameter, because such a large outer scale affects the tip/tilt over the telescope aperture, which is removed from the slopes before computing the correlations. Therefore, the estimated size of the outer scale may be biased to 2-3 times larger than the telescope diameter. ${ }^{12}$ In order to conclude that our result is biased or not, we need further studies by methods with a large spatial scale at Maunekea.

We can see roughly the same trend between the vertical profile of $C_{N}^{2}$ from RAVEN and the CFHT, and there is a strong ground layer over the night. When there is a difference in the total seeing between RAVEN and the CFHT, we can see the difference in the ground layer on the $C_{N}^{2}$ vertical profile, which may be because the Subaru and CFHT are located at different places and they have different dome seeings and local ground layers. Also, there is some differences at high altitudes. However, it is difficult to conclude what causes this difference, because each system observes a different direction, and each method has a different altitude resolution.

Fig.3 shows the histogram of the seeing. Here, we define the lower turbulence, which is lower than $1.5 \mathrm{~km}$, the upper turbulence, which is upper than $1.5 \mathrm{~km}$, the total turbulence, which is a sum of the lower and upper turbulence, and the unsensed turbulence, which is upper than $h_{\max }$ and included in the upper turbulence. The mean seeing values, mean $C_{N}^{2}$ fraction and mean $\mathcal{L}_{0}$ are summarized in Table 1 . The mean total seeing during the RAVEN on-sky observations is 0.475 arcsec, which is quite good condition compared with the nominal seeing. ${ }^{13}$ The lower turbulence contribution is $55 \%$ of the total turbulence, and $13 \%$ of the turbulence is unsensed by the cross-correlation. This unsensed turbulence is not taken into account in the tomographic reconstruction and one of the source causing the tomographic error.

Fig.4 shows the mean vertical profiles of $C_{N}^{2}$ and the median vertical profile of $\mathcal{L}_{0}$. The mean $C_{N}^{2}$ profile from the RAVEN SLODAR (black circles) has a strong turbulence at altitude lower than $1.5 \mathrm{~km}$ and weak turbulences at from $8 \mathrm{~km}$ to $16 \mathrm{~km}$. These features are consistent with the profile estimated by the CFHT DIMM and MASS (red triangles). The $C_{N}^{2}$ profile from the RAVEN SLODAR has also good agreement with the $C_{N}^{2}$ profile with

\begin{tabular}{cccc}
\hline & Mean Seeing $[\operatorname{arcsec}]$ & Mean $C_{N}^{2}$ fraction $[\%]$ & Mean $\mathcal{L}_{0}[\mathrm{~m}]$ \\
\hline Total Turbulence & 0.475 & 100 & 23 \\
Lower Turbulence & 0.327 & 55 & 17 \\
Upper Turbulence & 0.289 & 45 & 28 \\
Unsensed Turbulence & 0.124 & 13 & 37 \\
\hline
\end{tabular}

Table 1. The mean seeing, mean $C_{N}^{2}$ and $\mathcal{L}_{0}$ for the total, lower, upper and unsensed turbulence. 

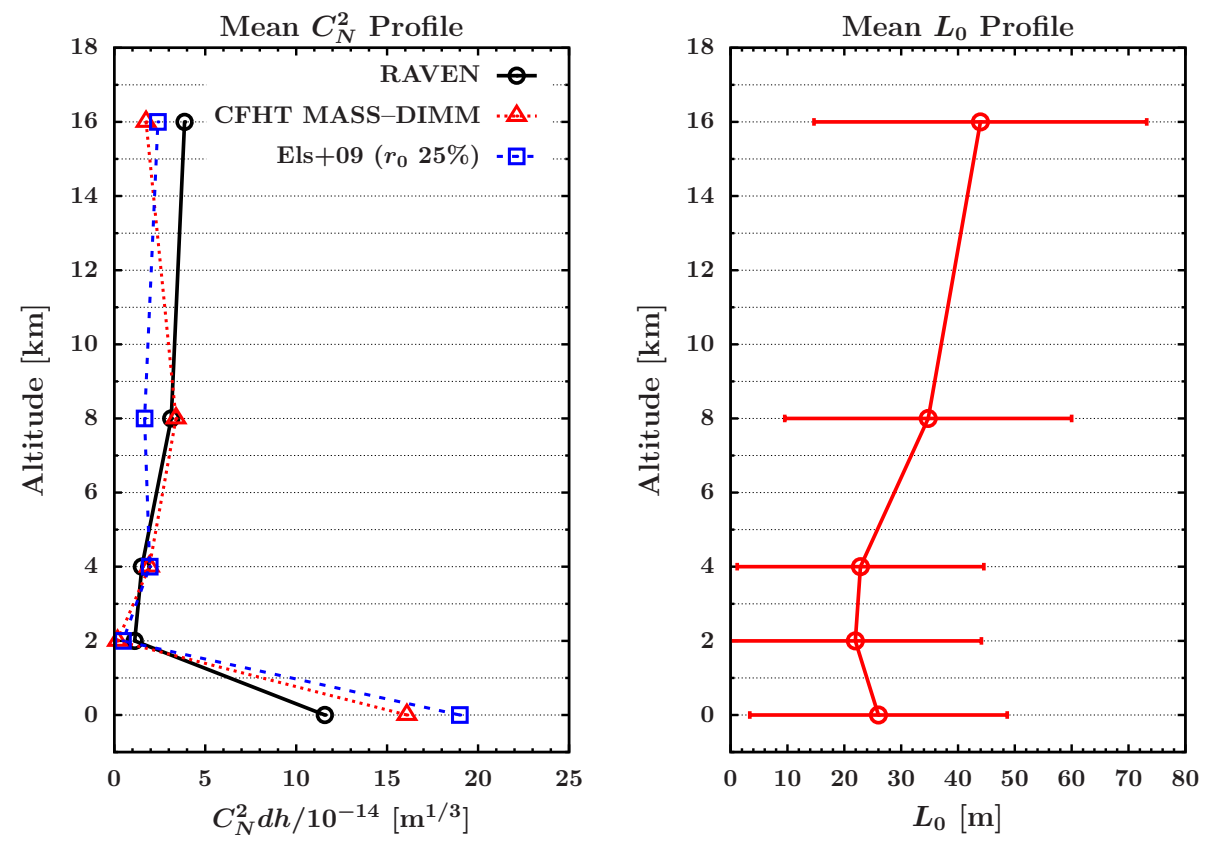

Figure 4. Left: Mean $C_{N}^{2}$ profile from the RAVEN SLODAR(red circles), the CFHT MASS and DIMM (green triangle), and the site characterization for the TMT reported in Els et al. (2009) (blue square). Right: Median $L_{0}$ profile estimated by the RAVEN SLODAR. The errorbars represent the scatter of the measurements.

$25 \%$-ile $r_{0}$ from the site characterization for the thirty meter telescope (TMT) reported in Els et al. (2009) ${ }^{13}$ (blue squares).

In the $\mathcal{L}_{0}$ profile, $\mathcal{L}_{0}$ value increases with altitude from $17 \mathrm{~m}$ at the ground to $37 \mathrm{~m}$ at $16 \mathrm{~km}$, and the similar profiles have been reported at several sites. ${ }^{8,14}$ One of the possibility to explain this trend is that, since the meta-pupil size increases with altitude, the SLODAR method is more sensitive to the large $\mathcal{L}_{0}$ compared with the lower altitudes. However, the $\mathcal{L}_{0}$ has large variations and it is difficult to conclude whether this profile is physically correct.

\section{CONCLUSION}

We present the statistics of vertical profiles of $C_{N}^{2}$ and the outer scale $\mathcal{L}_{0}$ at Maunakea estimated from 60 hours telemetry in total of multiple SH-WFSs of RAVEN by the fitted-SLODAR method. The mean seeing during the RAVEN on-sky observations is 0.475 arcsec, and $55 \%$ turbulence is below $1.5 \mathrm{~km}$. The mean outer scale is $23 \mathrm{~m}$, but, in order to conclude that our result is biased or not, we need further studies by methods with a large spatial scale at Maunekea. There is the unsensed turbulence higher than the maximum altitude measured by the SLODAR method, which is $13 \%$ total turbulence. The vertical profile of $C_{N}^{2}$ from the RAVEN SLODAR shows the strong turbulence at altitudes lower than $1.5 \mathrm{~km}$ and weak turbulence at altitudes higher than $8 \mathrm{~km}$, and has good agreements with the profile from CFHT DIMM and MASS, and TMT site characterization. The median $\mathcal{L}_{0}$ increases with altitude from $17 \mathrm{~m}$ at the ground to $37 \mathrm{~m}$ at $16 \mathrm{~km}$. However the outer scale profile has a strong variation.

\section{Acknowledgement}

The research leading to these results received the support of the JSPS Grant-in-Aid for JSPS Fellows (15J02510), the JSPS Grant-in- Aid for Young Scientist (B) (23740140), the JSPS Grant-in-Aid for Scientific Research (B) (26287027) and the A*MIDEX project (no. ANR-11-IDEX-0001-02) funded by the Investissements d'Avenir French Government program, managed by the French National Research Agency (ANR). 


\section{REFERENCES}

[1] Ono, Y. H. et al., "Multi time-step wavefront reconstruction for tomographic adaptive-optics systems," $J$. Opt. Soc. Am. A 33, 726-740 (Apr 2016).

[2] Ono, Y. H. et al., "On-sky MOAO performance evaluation of RAVEN," these proceeding, 9909-38 (2016).

[3] Martin, O. et al., "PSF reconstruction validated using on-sky CANARY data in MOAO mode," these proceeding , 9909-64 (2016).

[4] Neichel, B. et al., "Review on AO real-time turbulence estimation," these proceeding , 9909-16 (2016).

[5] Wilson, R. W., "SLODAR: measuring optical turbulence altitude with a ShackHartmann wavefront sensor," Monthly Notices of the Royal Astronomical Society 337(1), 103-108 (2002).

[6] Butterley, T. et al., "Determination of the profile of atmospheric optical turbulence strength from SLODAR data," Monthly Notices of the Royal Astronomical Society 369(2), 835-845 (2006).

[7] Cortés, A. et al., "Atmospheric turbulence profiling using multiple laser star wavefront sensors," Monthly Notices of the Royal Astronomical Society 427(3), 2089-2099 (2012).

[8] Guesalaga, A. et al., "Online estimation of atmospheric turbulence parameters and outer-scale profiling," these proceeding , 9909-134 (2016).

[9] Lardière, O. et al., "Multi-object adaptive optics on-sky results with Raven," Proc. SPIE 9148, 9148-14 (2014).

[10] Ziad, A. et al., "Comparison of measurements of the outer scale of turbulence by three different techniques," Appl. Opt. 43, 2316-2324 (Apr 2004).

[11] Maire, J. et al., "Wavefront outer scale deduced from interferometric dispersed fringes," $A \& A A 448(3)$, 1225-1234 (2006).

[12] Martin, O. et al., "William Herschel Telescope site characterization using the MOAO pathfinder CANARY on-sky data," these proceeding , 9909-163 (2016).

[13] Els, S. G. et al., "Thirty Meter Telescope Site Testing VI: Turbulence Profiles," Publications of the Astronomical Society of the Pacific 121(879), 527 (2009).

[14] Maire, J. et al., "Measurements of profiles of the wavefront outer scale using observations of the limb of the Moon," Monthly Notices of the Royal Astronomical Society 377(3), 1236-1244 (2007). 